\title{
Appropriateness and delay to initiate therapy in ventilator-associated pneumonia
}

\author{
C.M. Luna*, P. Aruj*, M.S. Niederman\#, J. Garzón*, D. Violi*, A. Prignoni*, F. Ríos`, \\ S. Baquero* and S. Gando*, for the Grupo Argentino de Estudio de la Neumonía \\ Asociada al Respirador (GANAR) group
}

ABSTRACT: Inappropriate therapy (IT) and delayed initiation of appropriate therapy (DIAT) result in inadequate therapy in patients with ventilator-associated pneumonia (VAP). The aim of the current study was to assess the impact of DIAT in VAP. A total of 76 mechanically ventilated patients with bacteriologically confirmed VAP were prospectively evaluated in the intensive care unit of six hospitals in Buenos Aires, Argentina.

Appropriate therapy was defined as coverage of all the identified pathogens by the antimicrobial therapy administered at the time of VAP clinical diagnosis. The clinical pulmonary infection score was measured during the 3 days before, at the onset and during the days which followed the onset of VAP. A total of 24 patients received adequate therapy; mortality was $29.2 \%$. The remaining 52 patients received either IT $(n=16)$ or DIAT $(n=36)$; the mortality was $63.5 \%$ combined, and $\mathbf{7 5 . 0}$ and $\mathbf{5 8 . 3 \%}$ for IT and DIAT, respectively (statistically significant compared with adequate therapy).

Inappropriate therapy and delayed initiation of appropriate therapy increased the mortality of ventilator-associated pneumonia. Patients with inappropriate therapy and/or delayed initiation of appropriate therapy had a more gradual increase in clinical pulmonary infection score than those receiving adequate therapy, and this increase was found to occur prior to the time of the clinical diagnosis.

In conclusion, these findings might provide the rationale for a trial of earlier initiation of therapy, based on clinical grounds in an effort to improve the outcome of patients with ventilatorassociated pneumonia.

KEYWORDS: Adequate therapy, antimicrobials, delayed therapy, nosocomial pneumonia, outcome, resistant pathogens

$\mathbf{V}$ entilator-associated pneumonia (VAP) is a frequent infectious complication among patients admitted to the intensive care unit (ICU), increasing the risk of death [1]. Outcome studies indicate that there are several factors related to mortality in VAP [2-6]. Delay in the initiation of therapy in pneumonia increases the risk of death in both community-acquired and nosocomial pneumonia $[7,8]$. The clinical pulmonary infection score (CPIS) was described to improve the clinical diagnosis of VAP, by including several criteria to quantify evidence of pneumonia [9]. CPIS has been used to evaluate the response to therapy, to select patients for a short course of antibiotics and to predict VAP outcome, when performed in a serial fashion [7, $8,10-12]$. Even though the sensitivity of the CPIS identification for bacteriologically confirmed VAP has been found to be $\geqslant 90 \%$ [9], it has a low diagnostic accuracy and is not superior or more accurate than the clinical prediction $[13,14]$. In one study, patients with a CPIS $\leqslant 6$ were treated successfully with only 3 days of antibiotic therapy [10]. These findings raised the question as to whether such a short duration was possible time possible, or whether some of these patients actually did not have VAP. If the former explanation is correct, then patients may benefit from early recognition and therapy of VAP.

Therefore, it was hypothesised that serial measurements of the CPIS in mechanically ventilated patients could be used, more precisely, to define the optimum time to start therapy for pneumonia patients in comparison with the use of standard clinical diagnosis. Taking into account the antibiotic susceptibility data for patients with VAP to define appropriate therapy (AT), measurements were taken of how often patients received $\mathrm{AT}$, because of the initiation of therapy at the earliest
AFFILIATIONS

*Pulmonary and Critical Care Divisions, Dept of Medicine, Hospital de Clínicas José de San Martín, Universidad de Buenos Aires, and - Critical Care Division, Policlínico Alejandro Posadas, Haedo, Provincia de Buenos Aires, Argentina. \# Dept of Medicine and Pulmonary and Critical Care Division, Winthrop University Hospital, Mineola NY, USA.

CORRESPONDENCE

C.M. Luna

Acevedo 1070

Banfield

1828

Buenos Aires

Argentina

Fax: 541142026068

E-mail: cymluna@fmed.uba.ar

Received:

April 232005

Accepted after revision:

August 122005 
and how often such therapy was initiated in a delayed fashion, using the CPIS as a sensitive tool to define the best time to start therapy of VAP. Knowing more clearly the number of patients receiving inappropriate therapy (IT) or delayed initiation of appropriate therapy (DIAT), the impact of therapy given in this manner was defined.

\section{METHODS}

\section{Study design and study subjects}

A prospective observational cohort study, conducted in the ICU of six hospitals from September 1999 to August 2003, was carried out. The inclusion criteria for subjects were to be aged $\geqslant 18 \mathrm{yrs}$ and have an estimated length of ventilation $>72 \mathrm{~h}$. Exclusion criteria were: admission after referral from another ICU; nosocomial pneumonia at the time of admission; and an AIDS-defining condition or neutropaenia $\left(<1,000 \cdot \mathrm{mm}^{-3}\right)$. The ethics committees from the participating institutions approved the study.

The data collected included the following: reason for admission; past medical history; vital signs; laboratory data; and chest radiographs, allowing for a calculation of a previously described modified version of the CPIS [9]. CPIS was calculated every day from the onset of mechanical ventilation up to the patient's discharge or death. In this modified CPIS (table 1), a tracheal aspirate culture was excluded as it was not available in time; leukocyte categories were reduced to two $(>5 \%$ of band forms were not considered because this information was not always available); and three categories instead of two were used for the aspect of sputum, to obtain a maximum of 10 points for the CPIS score [12]. The CPIS was calculated by one of two members of the investigation team

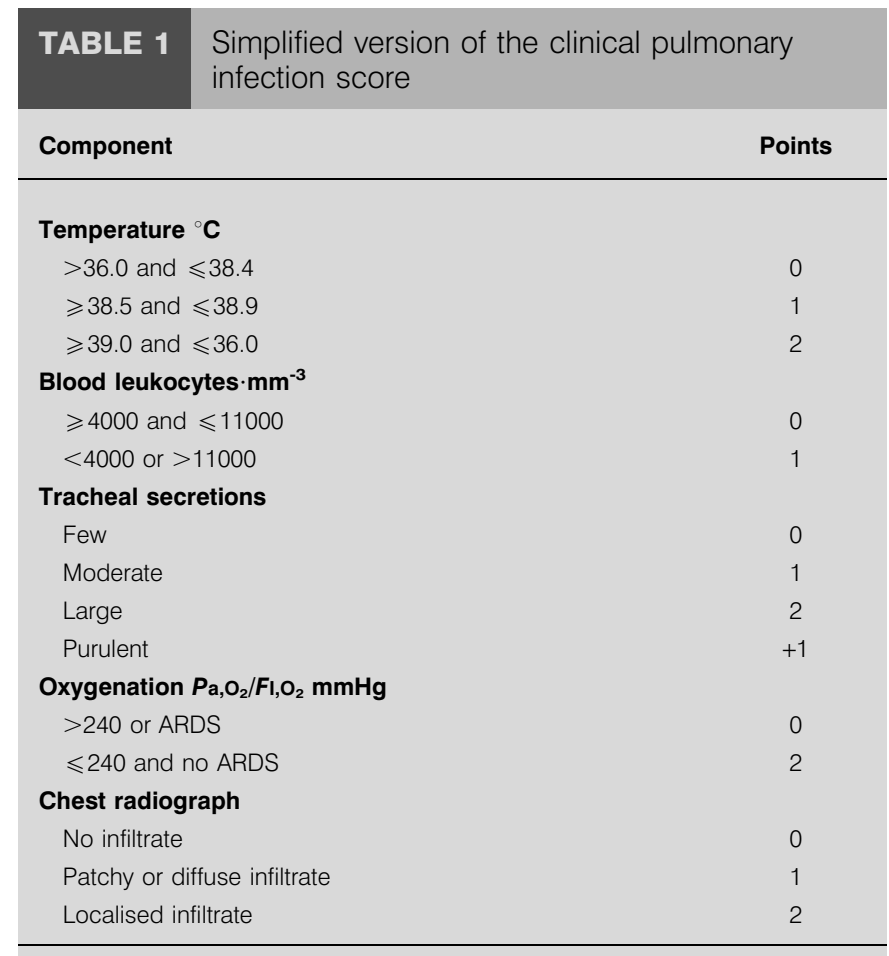

Total points for CPIS varied from 1-10 points. $\mathrm{Pa}_{1} \mathrm{O}_{2}$ : arterial oxygen tension; $\mathrm{Fl}, \mathrm{O}_{2}$ : inspiratory oxygen fraction; ARDS: acute respiratory disease syndrome.
(C.M. Luna or F. Ríos). Verification of data was performed before processing.

Patients were evaluated daily for evidence of VAP. CPIS and data on antimicrobial therapy were recorded. In patients with a clinical diagnosis of VAP, blood and bronchoalveolar lavage (BAL) fluid cultures were performed. For patients not developing VAP, the study ceased when they were weaned from mechanical ventilation.

A time-dependent analysis of the CPIS score was conducted for patients developing VAP. The time points were as follows: 3,2 , and 1 days before the onset of VAP (VAP-3, -2 and -1 ); the onset of VAP (VAP); and 3-7 days after the onset of VAP $(\mathrm{VAP}+3,+5$ and +7$)$.

VAP patients were followed daily until pneumonia was cured (discontinuation of antimicrobials or 21 days of antimicrobial therapy, whichever happened first), hospital discharge or death.

\section{Definitions}

Pneumonia was defined as new and persistent radiographical infiltrate(s) plus two of the following: 1) body temperature $>38^{\circ} \mathrm{C}$ or $<36^{\circ} \mathrm{C}$; 2) white blood cells $>10,000$ or $<4,000 \cdot \mathrm{mm}^{-3}$; or 3) macroscopically purulent tracheal aspirate [4]. Additionally, it was required that patients have microbiological confirmation, i.e. by growth of $\geqslant 10^{4}$ colony forming units $(\mathrm{cfu}) \cdot \mathrm{mL}^{-1}$ taken from a bronchoscopic BAL culture or isolation of a potential pathogen from the blood culture (unrelated to another source), and prescription of antimicrobials for pneumonia, after the clinical diagnosis.

The definition for the day of onset of VAP was the day that the attending physicians made the clinical diagnosis and prescribed antimicrobials for VAP, in patients fulfilling the clinical criteria of VAP.

Prior antimicrobial treatment was declared if there was current use of antimicrobials at the time of diagnosis of VAP, or previous use for $>24 \mathrm{~h}$ during the 10 days prior to VAP diagnosis. Indications for prior antimicrobials are listed in table 2 .

Appropriate therapy was defined as coverage of all the pathogens isolated (from blood or $\geqslant 10^{4} \mathrm{cfu} \cdot \mathrm{mL}^{-1}$ on BAL culture), by the antimicrobial therapy administered at the onset of VAP, determined by the sensitivity pattern in the antibiogram. AT could be adequate or not according to definitions published elsewhere [15].

Inadequate therapy involved not only IT (lack of coverage of all the pathogens isolated), but also DIAT. DIAT was defined as being present if AT was given within $24 \mathrm{~h}$ of the clinical diagnosis of VAP but also if the patient had a CPIS $\geqslant 5$ on the day before the clinical diagnosis was made (VAP-1). This definition was chosen as all of these VAP episodes were microbiologically defined, and if the CPIS had been calculated according to PUGIN et al. [9], they would have received two more points for a positive respiratory culture, giving a score of 7 , which is consistent with the presence of VAP. This definition allowed the current authors to test the hypothesis that these patients actually had VAP that was present before the time that it was recognised by standard clinical criteria, and that they 


\begin{tabular}{|c|c|c|c|}
\hline TABLE 2 & \multicolumn{3}{|c|}{$\begin{array}{l}\text { Indications for antimicrobial therapy in the } 57 \\
\text { patients on prior antibiotics at the time of the } \\
\text { clinical diagnosis of ventilator-associated } \\
\text { pneumonia (VAP) }\end{array}$} \\
\hline \multicolumn{2}{|c|}{ Indications for antimicrobials } & Cases n & $\begin{array}{l}\text { Duration of therapy at } \\
\text { VAP diagnosis days }\end{array}$ \\
\hline \multicolumn{4}{|c|}{ Community-acquired respiratory } \\
\hline \multicolumn{2}{|l|}{ Sepsis } & 16 & $5.8 \pm 2.9$ \\
\hline \multicolumn{4}{|c|}{ Prophylaxis before and/or after } \\
\hline \multicolumn{2}{|c|}{ surgery $>24 \mathrm{~h}$ or current } & 9 & $4.8 \pm 3.2$ \\
\hline \multicolumn{2}{|l|}{ Peritonitis } & 2 & $9.0 \pm 1.4$ \\
\hline \multicolumn{2}{|c|}{ Miscellaneous } & 10 & $5.1 \pm 5.2$ \\
\hline
\end{tabular}

might have benefited from earlier therapy. Thus, the inadequate therapy group included those with treatment delays due to a lack of sensitivity of the clinical diagnosis compared to the CPIS (DIAT), and those whose initial empirical antimicrobial treatment provided a lack of coverage of the aetiological pathogen (IT).

\section{Outcome criteria}

Mortality at day 28 after the VAP onset during hospitalisation was the main outcome criteria used. The length of stay and the days on antimicrobial therapy for VAP in survivors were also observed.

\section{Statistical analysis}

Continuous variables are expressed as mean \pm SEM, unless stated otherwise. Statistical significance was determined using an unpaired t-test. Differences between patients receiving adequate therapy and those receiving IT or DIAT were tested by two-way repeated measures ANOVA, and post hoc comparisons at specific time points were made by t-test with Bonferroni's correction. The Fisher's exact test was used to compare categorical variables. Significance was accepted for p-values $<0.05$.

\section{RESULTS}

During the period of study, 508 mechanically ventilated patients were admitted into the study. A total of 76 patients developed criteria for VAP (fulfilling two out of the three clinical criteria, plus the presence of a new or progressive infiltrate on the chest radiograph and microbiological confirmation). BAL microbial culture confirmed pulmonary infection in 72 out of the 76 patients and the blood culture in 16 of the 76 patients. In 10 cases, the pathogen isolated in the blood was not present in the BAL culture (in six cases it was a second pathogen, and in four cases the pathogens in the blood culture were the only isolates). Aetiological organisms are displayed in table 3 . In 15 cases, more than one pathogen was isolated (92 pathogens for 76 cases, i.e. 1.21 pathogens per case). The incidence rate of pneumonia was 14.9 per 100 mechanically ventilated patients.

A total of 58 patients $(76.3 \%)$ had received antibiotics prior to the development of VAP (mean \pm SD $5.0 \pm 2.9$, range 1-10 days):

\begin{tabular}{|c|c|c|c|c|c|}
\hline \multirow{3}{*}{$\begin{array}{l}\text { TABLE } 3 \\
\text { Pathogen }\end{array}$} & \multicolumn{5}{|c|}{$\begin{array}{l}\text { Number of isolates, adequacy of therapy and } \\
\text { mortality in the adequately treated and } \\
\text { inadequately treated patients }\end{array}$} \\
\hline & & \multicolumn{2}{|c|}{ IT-DIAT } & \multicolumn{2}{|c|}{ Adequate therapy } \\
\hline & & Total & Died & Total & Died \\
\hline \multicolumn{2}{|c|}{ Acinetobacter spp \# } & 19 & $13(68)$ & 7 & $3(43)$ \\
\hline \multicolumn{2}{|c|}{ Pseudomonas aeruginosa } & 17 & $12(71)$ & 2 & $1(50)$ \\
\hline \multicolumn{2}{|c|}{ Klebsiella pneumoniae } & 2 & $1(50)$ & 3 & $2(67)$ \\
\hline \multicolumn{2}{|c|}{ Enterobacter cloacae } & 2 & $2(100)$ & & \\
\hline \multicolumn{2}{|c|}{ Escherichia coli } & & & 2 & $0(0)$ \\
\hline \multicolumn{2}{|c|}{ Haemophilus influenzae } & & & 1 & $0(0)$ \\
\hline \multicolumn{6}{|c|}{ Staphylococcus aureus } \\
\hline \multicolumn{2}{|l|}{ MSSA } & 3 & $3(100)$ & 3 & $0(0)$ \\
\hline \multicolumn{2}{|l|}{ MRSA $^{\#}$} & 13 & $6(46)$ & 6 & $2(33)$ \\
\hline \multicolumn{2}{|c|}{ Streptococcus pneumoniae } & 2 & $0(0)$ & 2 & $0(0)$ \\
\hline \multicolumn{2}{|c|}{$\begin{array}{l}\text { Coagulase-negative } \\
\text { staphylococcus }\end{array}$} & 3 & $1(33)$ & & \\
\hline \multicolumn{2}{|c|}{ Corynebacterium spp } & 1 & $0(0)$ & & \\
\hline \multicolumn{2}{|l|}{ Candida spp } & 2 & $2(100)$ & & \\
\hline \multicolumn{2}{|c|}{ Other pathogens } & 2 & $0(0)$ & & \\
\hline
\end{tabular}

Data are presented as $\mathrm{n}$ or $\mathrm{n}(\%)$. In 76 ventilator-associated pneumonia patients, ninety-two isolates of causative microorganisms (65 multiresistant pathogens) were detected by bronchoalveolar lavage (BAL; $n=82$ ) and/or blood cultures $(n=16$, in six cases also isolated from the BAL; there was a second pathogen in six, and in four cases the pathogen in the blood was the only isolate). IT: inappropriate therapy; DIAT: delayed initiation of appropriate therapy; MRSA: methicillin-resistant $S$. aureus; MSSA: methicillin-sensitive $S$. aureus. ${ }^{\#}$ : multiresistant pathogens.

21 for a previous community-acquired lower-respiratory tract infection and 37 for an extrapulmonary infection. After the diagnosis of VAP, all patients received antibiotics; the total time of antibiotic therapy for all survivors was mean $\pm \mathrm{SD}$ $14.2 \pm 5.6$ days.

\section{Serial evaluation of clinical pulmonary infection score}

A time-dependent analysis of the CPIS was conducted for the overall population and independently for the patients receiving adequate therapy and those receiving either IT or DIAT. During the days prior to the clinical diagnosis of VAP, the CPIS rose from VAP-3 to VAP $(5.0 \pm 0.3$ versus $6.7 \pm 0.2 ; \mathrm{p}<0.001)$; thereafter, during the following days, the CPIS fell from VAP to $\mathrm{VAP}+3(6.7 \pm 0.2$ versus $6.1 \pm 0.2 ; \mathrm{p}=0.028)$ in the population as a whole. Comparing adequate therapy $(n=24)$ with IT-DIAT $(n=52)$, the CPIS score was similar in both groups at the VAP-3 $(4.6 \pm 0.5$ versus $5.2 \pm 0.3 ; p=0.273)$ and VAP $(6.4 \pm 0.4$ versus $6.9 \pm 0.2 ; \mathrm{p}=0.209)$ time points. However, the CPIS was higher, and rose more gradually toward a peak value, in the IT-DIAT patients compared with the patients who had been given adequate therapy, at VAP-2 $(5.5 \pm 0.3$ versus $4.2 \pm 0.3 ; \mathrm{p}=0.008)$ and VAP-1 (6.1 \pm 0.2 versus $3.9 \pm 0.3 ; \mathrm{p}=0.005$; fig. 1$)$. Thus, two separate populations were identified: one with adequate therapy who had a sudden rise in CPIS at the same time as the clinical diagnosis of VAP and received AT in a timely fashion; and one with IT-DIAT, who had a gradual rise in CPIS prior to the clinical diagnosis of VAP and received IT-DIAT. 
After the VAP time point, the CPIS exhibited a similar behaviour independent of the adequacy of therapy: CPIS for adequate therapy and IT-DIAT was $6.2 \pm 0.4$ versus $6.1 \pm 0.2$ at $\mathrm{VAP}+3$ time point $(\mathrm{p}=0.879$; fig. 1$)$. CPIS was $5.8 \pm 0.4$ versus $5.8 \pm 0.3$ at $\mathrm{VAP}+5$ and $5.8 \pm 0.4$ versus $5.8 \pm 0.3$ at $\mathrm{VAP}+7$ time points ( $p=0.962$ and 0.883 , respectively; data not shown).

\section{Adequacy of therapy and mortality}

Twenty-four patients received adequate therapy and 52 inadequate therapy (including 16 that received IT and 36 that received DIAT). Resistance to the antimicrobials prescribed at the onset of VAP was more common in patients infected with Acinetobacter spp, Pseudomonas aeruginosa, and methicillinresistant Staphylococcus aureus (table 3). The current authors examined mortality in relation to whether the therapy at the time of clinical suspicion of VAP was considered adequate or IT-DIAT.

The overall mortality rate was $52.6 \%$. In the group receiving adequate therapy, the mortality rate was $29.2 \%$, whereas it was $63.5 \%$ for those receiving inadequate therapy $(75.0 \%$ for IT and $58.3 \%$ for DIAT) giving p-values of $0.007,0.009$ and 0.036 , respectively, when compared with the adequate therapy group (fig. 2). There were no differences in the following: age; Acute Physiology and Chronic Health Evaluation (APACHE II) at admission; reason for mechanical ventilation; pathogens; days on prior mechanical ventilation or time from VAP onset and weaning or death, between adequate therapy and IT-DIAT (tables 4 and 5).

\section{DISCUSSION}

Several studies have demonstrated that initial treatment of VAP with an appropriate antimicrobial regimen is associated with lower mortality rates [16-19]. Thus, prompt identification

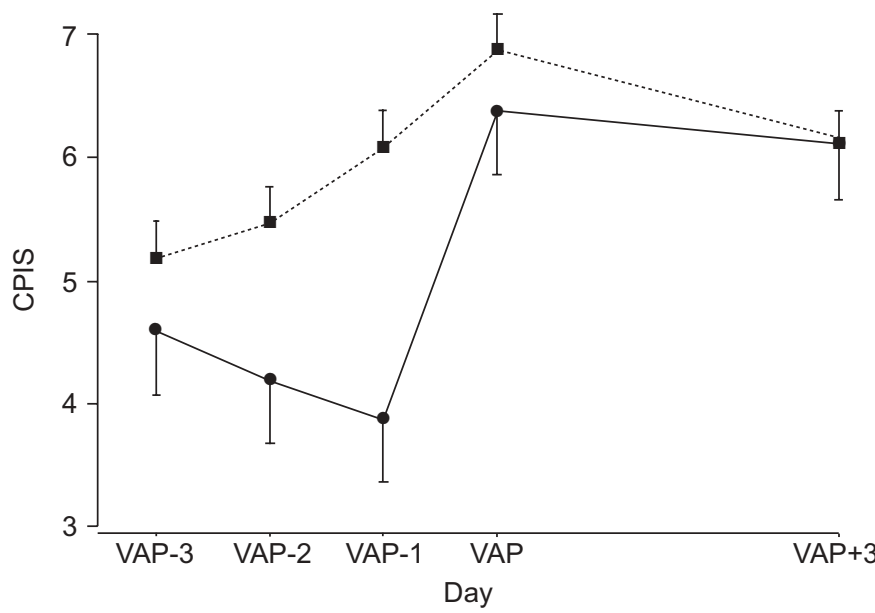

FIGURE 1. The clinical pulmonary infection score (CPIS) evolution between the ventilator associated pneumonia (VAP)-3 and the VAP+3 time points. There was a significant worsening at the VAP day compared with the CPIS 3 days before, then CPIS improved significantly during the following 3 days. The data were analysed by two-way repeated measures ANOVA. Post hoc analyses showed significant differences for the VAP-2 and VAP-1 time points, adequate therapy (-) versus inappropriate therapy-delayed initiation of appropriate therapy $(\boldsymbol{\square} ; \mathrm{p}=0.0083$ and 0.0051 , respectively). After VAP onset, the CPIS was the same at the VAP+3 time point for both groups.

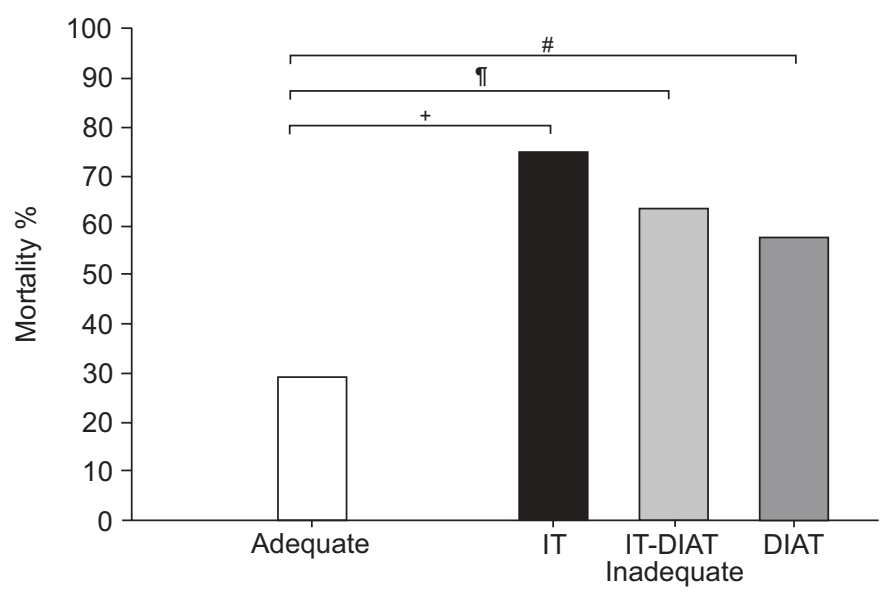

FIGURE 2. Statistical differences in the mortality rates observed between 24 (29.2\%) adequately and 52 (63.5\%) inadequately treated patients. The mortality rates of 16 (75\%) patients receiving inappropriate therapy (IT) and 36 (58.3\%) patients receiving delayed initiation of appropriate therapy (DIAT) are also displayed. ${ }^{\#}: p=0.036 ;{ }^{\bullet}: p=0.007 ;{ }^{+}: p=0.009$.

\section{TABLE 4 Reason for mechanical ventilation}

\begin{tabular}{|c|c|c|c|}
\hline $\begin{array}{l}\text { Reason for mechanical } \\
\text { ventilation }\end{array}$ & All & Adequate therapy & IT-DIAT \\
\hline $\begin{array}{l}\text { Post-operative } \\
\text { respiratory failure }\end{array}$ & 16 & 6 & 10 \\
\hline $\begin{array}{c}\text { Acute exacerbation of } \\
\text { chronic bronchitis }\end{array}$ & 9 & 2 & 7 \\
\hline Sepsis & 7 & 0 & 7 \\
\hline $\begin{array}{l}\text { Neurological or } \\
\text { neuromuscular disease }\end{array}$ & 4 & 2 & 2 \\
\hline $\begin{array}{l}\text { Community-acquired } \\
\text { pneumonia }\end{array}$ & 12 & 1 & 11 \\
\hline Coma & 9 & 3 & 6 \\
\hline Trauma & 7 & 3 & 4 \\
\hline Other ${ }^{\#}$ & 12 & 7 & 5 \\
\hline
\end{tabular}

of patients requiring treatment for VAP and careful selection of initial antibiotics are essential for the successful management of this life-threatening complication [17]. In the current study, it is confirmed that both inadequate therapy and delayed therapy are associated with a higher mortality rate than adequate therapy. However, it was observed that those who received adequate therapy had a sudden clinical deterioration that may have facilitated timely therapy, while those with a slower deterioration had a delay in therapy. The present authors propose, based on the findings in figure 1, to define clinical criteria for earlier initiation of therapy that could potentially lead to a reduction in VAP mortality.

The failure of initial antibiotic therapy due to the presence of resistant pathogens is one of the most important causes of poor 


\begin{tabular}{|c|c|c|c|c|}
\hline Characteristics & All & Adequate therapy & IT-DIAT & p-value \\
\hline Sex male/female & $48 / 28$ & $19 / 5$ & $29 / 23$ & 0.241 \\
\hline Age yrs & $63 \pm 17$ & $58 \pm 18$ & $66 \pm 17$ & 0.060 \\
\hline APACHE II score on admission to ICU & $19 \pm 6$ & $20 \pm 7$ & $19 \pm 6$ & 0.270 \\
\hline Time of mechanical ventilation VAP diagnosis days & $7.4 \pm 4.6$ & $7.4 \pm 4.8$ & $7.4 \pm 4.5$ & 0.966 \\
\hline Time between VAP diagnosis and weaning days & $8.2 \pm 6.0$ & $8.6 \pm 6.9$ & $8.0 \pm 6.2$ & 0.788 \\
\hline Time between VAP diagnosis and death days & $11.8 \pm 7.1$ & $10.9 \pm 5.1$ & $12.1 \pm 7.4$ & 0.901 \\
\hline Mortality & $40(52.6)$ & $7(29.2)$ & $33(63.5)$ & $0.007^{\#}$ \\
\hline
\end{tabular}

Data are presented as mean \pm SD or $n$ (\%), unless otherwise stated. IT: inappropriate therapy; DIAT: delayed initiation of appropriate therapy; APACHE II: Acute Physiology and Chronic Health Evaluation; ICU: intensive care unit. ${ }^{*}$ : statistically significant.

outcome [20, 21]. Adequacy of initial antimicrobial therapy is a major prognostic factor for patients with VAP. Thus, before new antibiotics are administered, reliable pulmonary specimens must be obtained for microbial examination [22]. In a previous study, a simplified CPIS score and its components were used to define the natural history VAP resolution and demonstrate that this approach can help to define early failure to response [12].

In the present study, the incidence of microbiologically confirmed VAP was $14.9 \%$. Similar to previous reports, approximately three-quarters of the patients involved in the study were on antibiotics at the time of VAP diagnosis [23, 24]. The patients were divided on the basis of the adequacy of initial empirical antibiotic therapy and the evolution of the CPIS from 3 days before, up to 7 days after the onset of VAP was examined. In 36 patients who received appropriate empirical antibiotic therapy after the clinical suspicion of $\mathrm{VAP}$, there was a delay in the initiation of therapy. This delay was defined as an appropriate treatment given within $24 \mathrm{~h}$ of the clinical diagnosis in patients who had a CPIS of $\geqslant 5$ for at least $24 \mathrm{~h}$ before the initiation of therapy. A CPIS of $\geqslant 5$ was chosen to define the correct time to start therapy, even if it occurred prior to when a clinical diagnosis was made. This was done as all these patients had VAP confirmed by BAL culture, and thus would have had a CPIS of $>6$ if microbiological criteria had been used, as originally described by PUGIN et al. [9]. The 36 patients with DIAT were pooled with the 16 patients who received initially empirical IT, and this IT-DIAT group consisted of all the patients who received inadequate antibiotic therapy.

It was observed (fig. 1) that at a time prior to the clinical diagnosis of VAP, the CPIS score was significantly higher at days VAP-2 and VAP-1 in the group of patients on IT-DIAT, compared with those receiving adequate therapy. It was also observed that there was no difference in the CPIS between the $\mathrm{VAP}$ and $\mathrm{VAP}+3,+5$ or +7 time points, when patients on adequate therapy were compared with those on IT-DIAT. However, the IT-DIAT group had a gradual rise in CPIS to a peak value, whereas those in the adequate therapy group had a sudden rise. Thus, those patients who had undergone a rapid clinical deterioration prior to the clinical diagnosis of VAP, as reflected by the CPIS, had timely initiation of therapy, possibly because the sudden change in clinical status facilitated the timely clinical recognition of pneumonia. However, for those with a more gradual rise in CPIS, it is believed that they all had $\mathrm{VAP}$ for $\geqslant 24 \mathrm{~h}$ prior to the clinical recognition of infection, and that the slow rise in CPIS, reflecting a more gradual deterioration, may have led to a delay in therapy for some. Since patients with adequate therapy had a better outcome than those with IT-DIAT, it is possible that earlier initiation of therapy could have had value for this latter group. In fact, when SINGH et al. [10] gave therapy to patients with a CPIS of $\leqslant 6$, they were able to treat patients for as few as 3 days, implying that early therapy can be more effective than delayed therapy. However, an alternative interpretation could be that following the CPIS criteria may be misleading, especially since the score improved at VAP-2 and VAP-1 in the adequately treated patients, and this may reflect a lack of sensitivity and specificity of CPIS to define the onset of pneumonia.

In a retrospective, cohort study on patients with ICU-acquired bacteraemic nosocomial infection or pneumonia, MATHEVON et al. [25] observed that the mortality rate was significantly associated with the length of time without appropriate antibiotic treatment $(p=0.011)$ and the number of organ failures on the day of diagnosis $(p=0.017)$. Appropriate antibiotic treatment only had an impact on survival if it was started within the first $24 \mathrm{~h}$ after sampling $(\mathrm{p}<0.02$ on day 0 and $<0.04$ on day 1 ). This difference remained after adjusting for the number of organ failures. This suggests that, during the course of nosocomial pneumonia and bacteraemia, the time at which appropriate antibiotic treatment is started is a key factor influencing survival [25]. This has also been demonstrated for S. aureus sepsis [26].

In a previous study, it was observed that when patients received antibiotics before the clinical suspicion of VAP, mortality was reduced if this previous therapy was appropriate, compared with when this therapy was inappropriate or if no therapy was given [4]. Changing from IT to AT, based on the results of BAL, did not reduce the mortality, as it was comparable with those who continued to receive IT, and this information became available too late to influence survival [4]. 
Thus, if AT was delayed, mortality was higher. In another study, it was observed that the combination of a nonimproving CPIS and inappropriate empirical therapy could identify a population that is unlikely to improve, regardless of changes in therapy, while this may not be true for patients who showed a favourable early response, in spite of IT [12]. In this prior study, a significant difference in the CPIS value between survivors and nonsurvivors during the 5 days following VAP onset was observed. Similar to the authors' prior study, in the current study, patients receiving IT-DIAT had a mortality of $77 \%$ if the CPIS remained $\geqslant 5$ during the 5 days after VAP onset, and $31 \%$ when the CPIS was $<5(\mathrm{p}=0.01)$, whereas in those receiving adequate therapy, mortality was $46 \%$ when the CPIS remained $\geqslant 5$ and $11 \%$ when CPIS was $<5(p=0.165$; data not shown).

Another recent study addressed the clinical importance of delays in the initiation of appropriate antibiotic treatment for VAP [8]. The study by IREGUI et al. [8] found a statistically significant association between the delay in administration of AT and hospital mortality for patients with VAP. Multiple logistic regression analysis identified this delay, increasing severity of illness as measured by APACHE II scores and the presence of underlying malignancy as important determinants of hospital mortality.

The current findings, which related the adequacy of therapy to the time of initiating therapy, confirmed that early initiation of AT may improve the survival of patients with VAP and that a more sensitive tool than clinical diagnosis may be needed to define the optimal time to initiate therapy in an effort to alter the course of illness. The present study's data show that the most sensitive criterion for defining this time point may be when the modified CPIS (table 1) reaches $\geqslant 5$, which, in some patients, occurred even prior to the clinical diagnosis of VAP.

To summarise, the authors conducted a prospective evaluation of the natural history of ventilator-associated pneumonia during therapy in patients that received both adequate therapy and inadequate therapy. While other studies have looked at the adequacy of therapy at the time of clinical suspicion of ventilator-associated pneumonia, there is only one study that related the timing of the administration of appropriate therapy to the time of onset of symptoms [8], and, to the current authors' knowledge, this is the first study that has prospectively evaluated the evolution of the clinical pulmonary infection score and used these data to define delayed initiation of appropriate therapy. The current study's findings suggest that serial measurements of the clinical pulmonary infection score in mechanically ventilated patients could be used to identify patients developing pneumonia that was not yet clinically recognised. Since the mortality of patients with inappropriate therapy or delayed initiation of appropriate therapy differed from patients with adequate therapy, the authors believe that an earlier initiation of appropriate therapy, guided by the clinical pulmonary infection score or another clinical score, could lead to an improved outcome of patients with ventilator-associated pneumonia. However, this is still a hypothesis that could be tested in subsequent clinical trials to define the optimal time to initiate antibiotic therapy.

\section{ACKNOWLEDGEMENTS}

Members of the Grupo Argentino de Estudio de la Neumonía Asociada al Respirador (GANAR): C. Apezteguía (Policlínico Alejandro Posadas, Buenos Aires); P. Desmery (Sanatorio Mitre, Capital Federal); W. Matarucco (Sanatorio OtamendiMiroli, Buenos Aires); F. Pálizas (Clínica Bazterrica, Buenos Aires); and G. Menga (Hospital María Ferrer, Gobierno de la Ciudad, Buenos Aires).

\section{REFERENCES}

1 Vincent JL, Bihari DJ, Suter PM, et al. The prevalence of nosocomial infection in intensive care units in Europe. JAMA 1995; 274: 639-644.

2 Heyland DK, Cook DJ, Griffith L, Keenan SP, BrunBuisson C. The attributable morbidity and mortality of ventilator-associated pneumonia in the critically ill patient. Am J Respir Crit Care Med 1999; 159: 2249-2256.

3 Hernandez C, el-Ebiary M, Gonzalez J, de la Bellacasa JP, Monton C, Torres A. Relationship between ventilatorassociated pneumonia and intramucosal gastric pHi: a case-control study. J Crit Care 1996; 11: 122-128.

4 Luna CM, Vujacich P, Niederman MS, et al. Impact of BAL data on the therapy and outcome of ventilator-associated pneumonia. Chest 1997; 111: 676-685.

5 Bonten MJ, Bergmans DC, Stobberingh EE, et al. Implementation of bronchoscopic techniques in the diagnosis of ventilator-associated pneumonia to reduce antibiotic use. Am J Respir Crit Care Med 1997; 156: 1820-1824.

6 Kollef MH, Ward S. The influence of mini-BAL cultures on patient outcomes: implications for the antibiotic management of ventilator-associated pneumonia. Chest 1998; 113: 412-420.

7 Meehan TP, Chua-Reyes JM, Tate J, et al. Process of care performance, patient characteristics and outcomes in elderly patients hospitalized with community-acquired or nursing home-acquired pneumonia. Chest 2000; 117: 1378-1385.

8 Iregui M, Ward S, Sherman G, Fraser V, Kollef M. Clinical importance of delays in the initiation of appropriate antibiotic treatment for ventilator-associated pneumonia. Chest 2002; 122: 262-268.

9 Pugin J, Auckenthaler R, Mili N, Janssens JP, Lew PD, Suter PM. Diagnosis of ventilator-associated pneumonia by bacteriologic analysis of bronchoscopic and nonbronchoscopic "blind" bronchoalveolar lavage fluid. Am Rev Respir Dis 1991; 143: 1121-1129.

10 Singh N, Rogers P, Atwood CW, Wagener MM, Yu VL. Short-course empiric antibiotic therapy for patients with pulmonary infiltrates in the intensive care unit. A proposed solution for indiscriminate antibiotic prescription. Am J Respir Crit Care Med 2000; 162: 505-511.

11 Garrard CS, A'Court CD. The diagnosis of pneumonia in the critically ill. Chest 1995; 108: Suppl. 2, 15S-25S.

12 Luna CM, Blanzaco D, Niederman MS, et al. Resolution of ventilator-associated pneumonia: prospective evaluation of the clinical pulmonary infection score as an early clinical predictor of outcome. Crit Care Med 2003; 31: 676-682.

13 Fabregas N, Ewig S, Torres A, et al. Clinical diagnosis of ventilator associated pneumonia revisited: comparative 
validation using immediate post-mortem lung biopsies. Thorax 1999; 54: 867-873.

14 Fartoukh M, Maitre B, Honore S, Cerf C, Zahar JR, BrunBuisson C. Diagnosing pneumonia during mechanical ventilation: the clinical pulmonary infection score revisited. Am J Respir Crit Care Med 2003; 168: 173-179.

15 Niederman MS, Craven DE, Bonten MJ, et al. Guidelines for the management of adults with hospital-acquired, ventilator-associated and healthcare-associated pneumonia. Am J Respir Crit Care Med 2005; 171: 388-416.

16 Kollef MH, Sherman G, Ward S, Fraser VJ. Inadequate antimicrobial treatment of infections: A risk factor for hospital mortality among critically ill patients. Chest 1999; 115: 462-474.

17 Alvarez-Lerma F. Modifications of empiric antibiotic treatment in patients with pneumonia acquired in the intensive care unit. ICU-Acquired Pneumonia Study Group. Intensive Care Med 1996; 22: 387-394.

18 Rello J, Rue M, Jubert $\mathrm{P}$, et al. Survival in patients with nosocomial pneumonia: impact of the severity of illness and the etiologic agent. Crit Care Med 1997; 25: 1862-1867.

19 Luna CM, Videla AJ, Matera J, et al. Blood cultures have limited value in predicting severity of illness and as a diagnostic tool in ventilator-associated pneumonia. Chest 1999; 116: 1075-1084.
20 Lowenkron SW, Niederman M. Definition and evaluation of the resolution of nosocomial pneumonia. Semin Respir Infect 1992; 7: 271-281.

21 American Thoracic Society. Hospital-acquired pneumonia in adults: diagnosis, assesment of severity, initial antimicrobial therapy, and preventive strategies. A consensus statement. Am J Respir Crit Care Med 1995; 153: 1711-1725.

22 Fagon JY, Chastre J. Diagnosis and treatment of nosocomial pneumonia in ALI/ARDS patients. Eur Respir J 2003; 21: Suppl. $42,77 \mathrm{~s}-83 \mathrm{~s}$.

23 Chastre J, Viau F, Brun P, et al. Prospective evaluation of the protected specimen brush for the diagnosis of pulmonary infections in ventilated patients. Am Rev Respir Dis 1984; 130: 924-929.

24 Chastre J, Fagon JY, Bornet-Lecso M, et al. Evaluation of bronchoscopic technique for the diagnosis of nosocomial pneumonia. Am J Respir Crit Care Med 1995; 152: 231-240.

25 Mathevon T, Souweine B, Traore O, Aublet B, Caillaud D. ICU-acquired nosocomial infection: impact of delay of adequate antibiotic treatment. Scand J Infect Dis 2002; 34: 831-835.

26 Falcone M, Carfagna P, Cassone M, et al. Staphylococcus aureus sepsis in hospitalized non neutropenic patients: retrospective clinical and microbiological analysis. Ann Ital Med Int 2002; 17: 166-172. 